\title{
Elevated Plasma Levels of Endostatin Are Associated with Chronic Kidney Disease
}

\author{
Jing Chen ${ }^{a-c}$ L. Lee Hamm ${ }^{a, b} \quad$ Myra A. Kleinpeter ${ }^{a} \quad$ Fred Husserl $^{d}$ \\ Islam Enver Khan ${ }^{a}$ Chung-Shiuan Chen ${ }^{c}$ Yanxi Liu ${ }^{c}$ Katherine T. Mills ${ }^{c}$ \\ Chuan He $^{c}$ Nader Rifai ${ }^{e}$ Eric E. Simon ${ }^{a}$ Jiang He ${ }^{a-c}$ \\ ${ }^{a}$ Department of Medicine and ${ }^{b}$ Tulane Hypertension and Renal Center of Excellence, Tulane University School of \\ Medicine, 'Department of Epidemiology, Tulane University School of Public Health and Tropical Medicine, and \\ ${ }^{d}$ Department of Nephrology, Ochsner Health System, New Orleans, La., and eDepartment of Pathology, Children's \\ Hospital Boston, Harvard Medical School, Boston, Mass., USA
}

\section{Key Words}

Albuminuria $\cdot$ Antiangiogenic factor $\cdot$ Chronic kidney disease $\cdot$ Endostatin $\cdot$ Estimated glomerular filtration rate

\begin{abstract}
Background/Aims: Angiogenesis may play an important role in the renal repair process after injury. We investigated the association between plasma endostatin, an endothelialspecific antiangiogenic factor, and chronic kidney disease (CKD). Methods: We compared plasma endostatin levels in 201 CKD patients and 201 controls. CKD was defined as estimated glomerular filtration rate (eGFR) $<60 \mathrm{ml} / \mathrm{min} / 1.73 \mathrm{~m}^{2}$ or presence of albuminuria ( $\geq 30 \mathrm{mg} / 24 \mathrm{~h}$ ). Results: After adjustment for established CKD risk factors, the median (interquartile range) of plasma endostatin was $276.7 \mathrm{ng} / \mathrm{dl}$ (199.3357.5) in patients with CKD and 119.4 ng/dl (103.7-134.6) in controls without CKD ( $p<0.0001$ for group difference). logtransformed plasma endostatin was significantly and inversely correlated with eGFR $(r=-0.83, p<0.0001)$ and positively correlated with log-transformed urine albumin $(r=$ $0.66, p<0.0001$ ) in the study participants. In addition, one standard deviation increase in log-transformed plasma endostatin $(0.55 \mathrm{ng} / \mathrm{dl})$ was associated with a decline in eGFR of $-26.2 \mathrm{ml} / \mathrm{min}$ and an increase in urine albumin of $3.26 \mathrm{mg} /$
\end{abstract}

$24 \mathrm{~h}$ after adjusting for multiple covariables. Furthermore, the multivariable-adjusted odds ratio for CKD comparing the highest tertile ( $\geq 131.4 \mathrm{ng} / \mathrm{dl}$ ) to the two lower tertiles of plasma endostatin was 21.6 (95\% Cl: 10.2-45.5; p < 0.0001). Conclusion: These data indicate that elevated plasma endostatin is strongly and independently associated with CKD. Prospective cohort studies and clinical trials are warranted to further examine the causal relationship between endostatin and risk of CKD and to develop novel interventions targeting circulating endostatin aimed at reducing CKD risk.

Copyright $\odot 2012$ S. Karger AG, Basel

\section{Introduction}

Chronic kidney disease (CKD) is prevalent worldwide and a major risk factor for end-stage renal disease, cardiovascular disease (CVD), and premature death [1]. Many traditional and emerging risk factors have been associated with the etiology of CKD [2]. Recently, it has been hypothesized that the progressive deterioration of the renal microvasculature and consequent tubulointerstitial fibrosis and glomerulosclerosis are involved in the development and progression of CKD $[3,4]$. Angiogenesis plays an important role in maintenance of the glomer-

\section{KARGER}

Fax +4161306 1234

E-Mail karger@karger.ch

www.karger.com
(C) 2012 S. Karger AG, Basel

0250-8095/12/0354-0335\$38.00/0

Accessible online at:

www.karger.com/ajn
Jing Chen, MD, MS

Department of Medicine, Tulane University School of Medicine

1430 Tulane Avenue SL45

New Orleans, LA 70112 (USA)

Tel. +1 504588 6110, E-Mail jchen@ tulane.edu 
ular capillary structure and filtration barrier and in the renal repair process after injury $[3,4]$. Angiogenesis is controlled by the balance between proangiogenic and antiangiogenic factors. Animal experiments and small clinical studies have suggested that proangiogenic factors, such as vascular endothelial growth factor (VEGF), may play an important role in the pathogenesis of CKD [5-8]. However, the role of antiangiogenic factors in the pathogenesis of CKD has not been well studied.

Endostatin, a 20-kDa C-terminal fragment of collagen XVIII, is one of the most potent endothelial cell-specific inhibitors of angiogenesis and has been shown to specifically influence proliferation, migration, and apoptosis of endothelial cells in vitro $[9,10]$. The inhibitory effects of endostatin on the expression of proangiogenic factors and vascular permeability have been reported [11]. Recently, experimental studies suggested that endostatin may lead to the rarefaction of renal microvasculature [12, 13]. On the other hand, an animal study has reported that glomerular hypertrophy, hyperfiltration, and albuminuria are significantly suppressed by treatment with endostatin peptide in the streptozotocin-induced diabetic nephropathy mouse model [14]. The effect of circulating endostatin levels on the risk of CKD has rarely been investigated in human subjects. In a published clinical study, Futrakul et al. [15] reported significantly increased circulating endostatin in $60 \mathrm{CKD}$ patients compared to 15 normal controls. In the present study, we investigated the association of plasma endostatin with CKD in 201 patients with and 201 controls without CKD.

\section{Methods}

\section{Study Participants}

We recruited $201 \mathrm{CKD}$ patients and 201 controls without CKD in the greater New Orleans, Louisiana area from 2007 to 2010. CKD patients aged 21-74 years were recruited from nephrology and internal medicine clinics via physician referral by trained research staff in the study area. All eligible CKD patients identified in the recruiting clinics were invited to participate in the study. CKD was defined as estimated glomerular filtration rate (eGFR) $<60 \mathrm{ml} / \mathrm{min} / 1.73 \mathrm{~m}^{2}$ or presence of albuminuria ( $\geq 30 \mathrm{mg} / 24 \mathrm{~h}$ ). Patients were excluded if they had a history of chronic dialysis, kidney transplants, immunotherapy in the past 6 months, chemotherapy within the past 2 years, and current clinical trial participation that may have an impact on CKD. Additional exclusion criteria were history of HIV or AIDS and inability or unwillingness to give informed consent. Controls were recruited through mass mailing to residents aged 21-74 years living in the same area according to zip code. The eligibility of controls was assessed by a prescreening telephone interview and a clinic screening visit. Individuals were included if they had no evidence of CKD (eGFR
$>60 \mathrm{ml} / \mathrm{min} / 1.73 \mathrm{~m}^{2}$ and no persistent albuminuria). Cases and controls were frequency-matched according to age group (10 years), gender, and race to increase the efficiency of patient recruitment and statistical analysis.

The Tulane University Internal Review Board approved this study, and informed consent was obtained at the screening visit from all study participants.

\section{Measurements}

A standard questionnaire was administered by trained staff at a clinical visit to obtain demographic information, lifestyle risk factors (including cigarette smoking, alcohol drinking, and physical activity), self-reported history of CVD, diabetes, hypercholesterolemia, and hypertension, as well as the use of antihypertensive, lipid-lowering, and antidiabetic medications.

Three blood pressure measurements were obtained at a clinical visit by trained and certified staff according to a common protocol adapted from procedures recommended by the American Heart Association [16]. A standard mercury sphygmomanometer was used, and one of four cuff sizes (pediatric, regular adult, large, or thigh) was chosen on the basis of the circumference of the participant's arm. Body height and weight were obtained by trained staff and used to calculate BMI and body surface area using Mosteller's formula [(weight in $\mathrm{kg} \times$ height in $\left.\mathrm{cm}) / 3,600)^{1 / 2}\right][17]$.

An overnight fasting blood sample was collected to measure plasma endostatin and glucose, serum creatinine (SCr) and cholesterol, and triglycerides. eGFR was estimated from SCr, sex, age, and race using the CKD-Epi equation: GFR $=141 \times \min (\mathrm{SCr} /$ $\kappa, 1) \alpha \times \max (\mathrm{SCr} / \kappa, 1)^{-1.209} \times 0.993^{\mathrm{Age}} \times 1.018$ (iffemale) $\times 1.159$ (if black), where $\kappa$ is 0.7 and 0.9 and $\alpha$ is -0.329 and -0.411 for females and males, respectively [18]. A 24-hour urinary sample was collected to measure creatinine and albumin.

Serum cholesterol and triglyceride levels were assayed using an enzymatic procedure on a Hitachi 902 automatic analyzer (Roche Diagnostics, Indianapolis, Ind., USA). Serum glucose was measured using the hexokinase enzymatic method (Roche Diagnostics). SCr was measured using the Roche enzymatic method (Roche-Hitachi P-Module instrument with Roche Creatininase Plus assay; Hoffman-La Roche, Basel, Switzerland). Urinary concentrations of albumin and creatinine were measured with a DCA 2000 Analyzer (Bayer AG, Leverkusen, Germany). Plasma endostatin was measured with ELISA assay kits from R\&D Systems. The assay employs the quantitative sandwich enzyme immunoassay technique, and all samples were assayed in duplicate. The intra-assay and interassay coefficients of variation were 3.6 and $5.7 \%$, respectively.

\section{Statistical Analysis}

Medians and interquartile ranges for plasma endostatin were calculated for CKD patients and controls, and the Mann-Whitney test was used to test differences in the unadjusted medians. Quantile regression was used to obtain adjusted medians (interquartile ranges) and the Wald test was used to test differences in the adjusted medians between CKD patients and controls [19]. Age, gender, race, high school education, current cigarette smoking, weekly alcohol consumption, physical activity ( $\geq$ twice per week), BMI, low-density lipoprotein (LDL) cholesterol, serum glucose, systolic blood pressure, history of hypertension, diabetes, CVD, and medication uses were adjusted in these analyses. 
Table 1. Characteristics of 201 patients with CKD and 201 controls

\begin{tabular}{|c|c|c|c|}
\hline Age, years & $55.9 \pm 9.9$ & $52.5 \pm 10.0$ & 0.0007 \\
\hline Males, \% & 55.2 & 45.3 & 0.056 \\
\hline Blacks, $\%$ & 60.7 & 51.2 & 0.06 \\
\hline High school education, $\%$ & 58.5 & 81.6 & $<0.0001$ \\
\hline $\mathrm{BMI}$ & $32.2 \pm 7.8$ & $28.9 \pm 6.4$ & $<0.0001$ \\
\hline Systolic blood pressure, $\mathrm{mm} \mathrm{Hg}$ & $132.2 \pm 21.0$ & $122.0 \pm 14.7$ & $<0.0001$ \\
\hline Diastolic blood pressure, $\mathrm{mm} \mathrm{Hg}$ & $77.2 \pm 13.5$ & $77.6 \pm 9.4$ & 0.77 \\
\hline Plasma glucose, $\mathrm{mg} / \mathrm{dl}$ & $119.9 \pm 46.7$ & $103.4 \pm 35.4$ & $<0.0001$ \\
\hline LDL-cholesterol, mg/dl & $103.3 \pm 45.9$ & $118.2 \pm 30.2$ & $<0.0001$ \\
\hline History of CVD, \% & 43.7 & 7.0 & $<0.0001$ \\
\hline $\mathrm{eGFR}, \mathrm{ml} / \mathrm{min} / 1.73 \mathrm{~m}^{2}$ & $43.3 \pm 19.9$ & $96.7 \pm 16.8$ & $<0.0001$ \\
\hline Urinary albumin, $\mathrm{mg} / 24 \mathrm{~h}^{\mathrm{a}}$ & $74.5(12.3-417.4)$ & $5.9(4.1-11.4)$ & $<0.0001$ \\
\hline Plasma endostatin, ng/dl ${ }^{\mathrm{a}}$ & $265.8(189.6-363.3)$ & $119.1(102.7-136.1)$ & $<0.0001$ \\
\hline
\end{tabular}

a Median (interquartile range).

Multivariable linear regression was used to examine the association of eGFR and plasma endostatin levels after adjustment for the previously mentioned covariates. log transformations were used for plasma endostatin and urinary albumin levels because they were not normally distributed. In addition, multivariate logistic regression was used to obtain adjusted odds ratios comparing the highest tertile of plasma endostatin levels to the lower two tertiles between CKD patients and controls. Plasma endostatin tertiles were defined based upon measurements in the control group. All analyses were performed using SAS version 9.2 statistical software (Cary, N.C., USA).

\section{Results}

The general characteristics of study participants by CKD status are presented in table 1. Those with CKD were older, less educated, heavier, and less likely to drink alcohol compared to those without CKD. In addition, they were more likely to have a history of CVD, hypertension, diabetes, and hypercholesterolemia. Mean BMI, systolic blood pressure, serum glucose, and urinary albumin were significantly higher, while LDL-cholesterol and eGFR were lower in CKD patients compared to controls.

The entire distribution of plasma endostatin was shifted to higher levels in CKD patients compared to that in controls (fig. 1). The median plasma endostatin level and

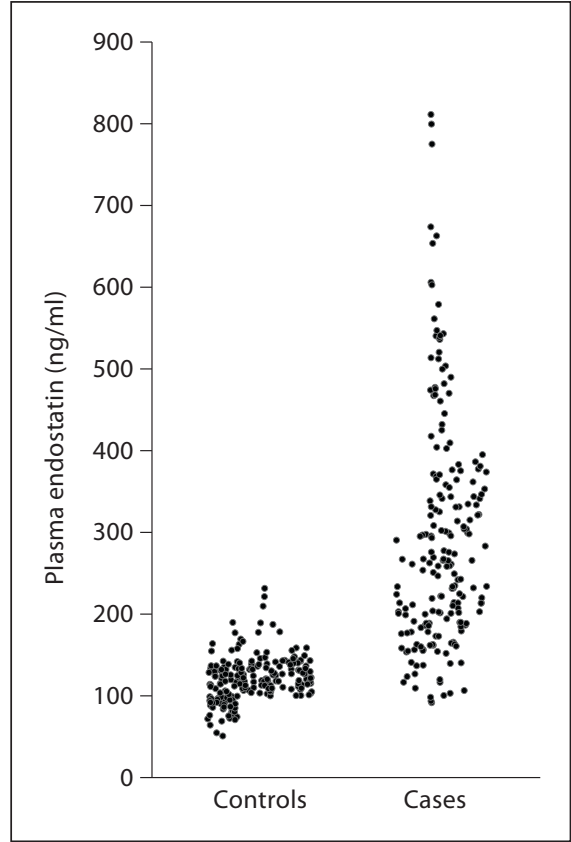

Fig. 1. Plots of plasma endostatin levels in $201 \mathrm{CKD}$ patients compared to 201 controls. 


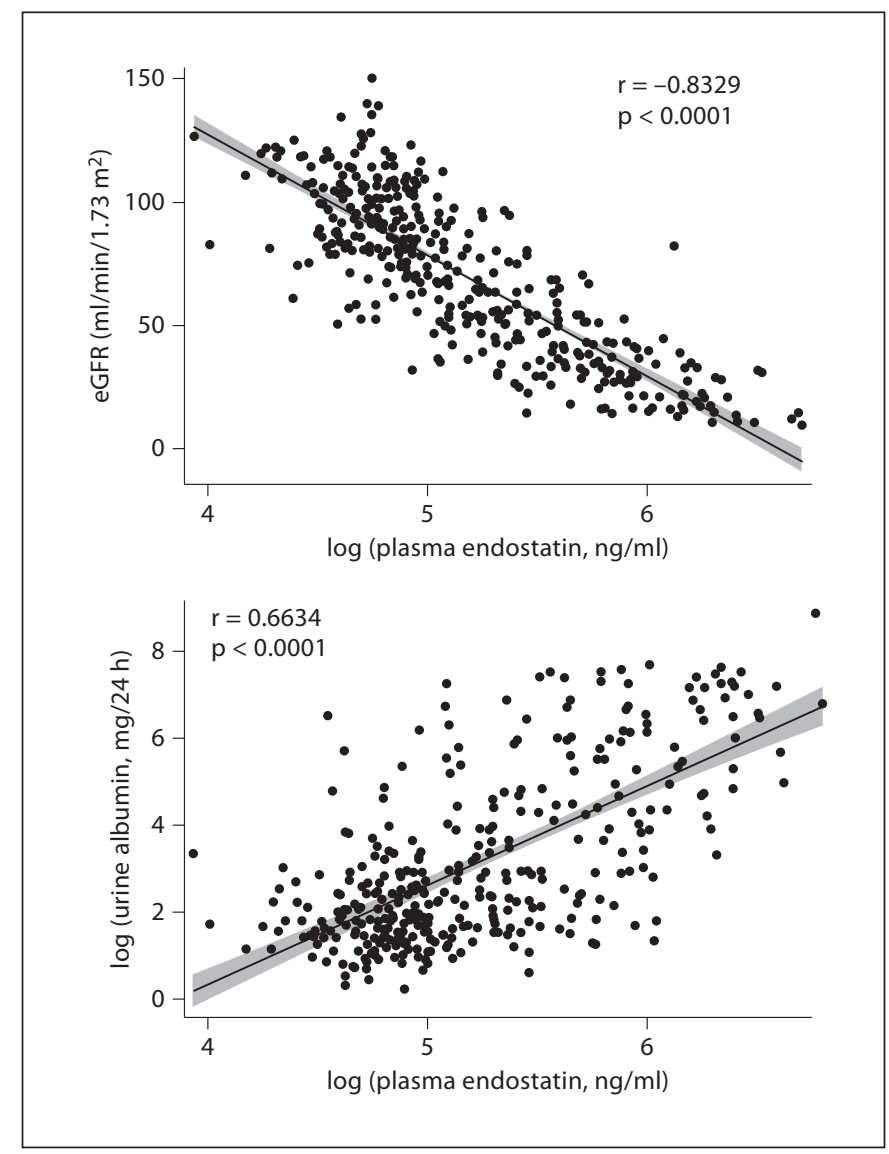

Fig. 2. Scatterplots of log translation of plasma endostatin levels and eGFR (upper panel) and log-transformed 24-hour urinary albuminuria excretions (lower panel) with fitted regression line. Shaded area represents $95 \%$ CI.

interquartile range was significantly higher in CKD patients $(265.8 \mathrm{ng} / \mathrm{ml}, 189.6-363.3)$ compared to controls (119.1 ng/ml, 102.7-136.1; $\mathrm{p}<0.0001$ for group difference). After adjustment for age, gender, race, high school education, physical activity, current cigarette smoking, weekly alcohol drinking, BMI, LDL-cholesterol, glucose, systolic blood pressure, and history of CVD, the median plasma endostatin level remained significantly higher in CKD patients $(276.7 \mathrm{ng} / \mathrm{ml}, 199.3-357.5)$ compared to controls (119.4 ng/ml, 103.7-134.6; $\mathrm{p}<0.0001$ for group difference).

The scatter plots of plasma endostatin levels versus eGFR and urinary albumin excretion show that plasma endostatin was significantly associated with the severity of CKD (fig. 2). log-transformed plasma endostatin levels were significantly and inversely correlated with eGFR $(r=-0.83, p<0.0001)$ and positively correlated with log- transformed urinary albumin excretion $(\mathrm{r}=0.66, \mathrm{p}<$ 0.0001 ). In the linear regression analyses adjusted for multiple covariables, the log-transformed plasma endostatin levels were significantly and inversely related to eGFR and positively related to log-transformed urinary albumin excretion (table 2). For example, one standard deviation increase in log-transformed plasma endostatin $(0.55 \mathrm{ng} / \mathrm{dl})$ was associated with a decline in eGFR of $-26.2 \mathrm{ml} / \mathrm{min}$ and an increase in urine albumin of 3.26 $\mathrm{mg} / 24 \mathrm{~h}$ (after back transformation) in the multivariable models.

In the logistic regression analyses adjusted for age, gender, and race, the participants in the highest tertile ( $\geq 67$ th percentile) of plasma endostatin had a significant 31-fold higher odds ratio of CKD compared to those in the lower two tertiles (table 3). After further adjustment for education, cigarette smoking, alcohol drinking, physical activity, BMI, LDL-cholesterol, systolic blood pressure, glucose, and history of CVD, the odds ratio of CKD associated with the top tertile of plasma endostatin was 21.6-fold higher compared to lower plasma endostatin. Furthermore, adjustment for the history of hypertension and diabetes did not significantly change odds ratio estimates.

\section{Discussion}

Our study showed that the plasma endostatin level was significantly higher in patients with CKD compared to controls without CKD. In addition, there was a strong dose-response and significant association between the plasma level of endostatin and the severity of CKD assessed by eGFR and albuminuria, independent from established risk factors for CKD including hypertension, diabetes, and CVD. These findings may have important clinical implications.

In recent years, the effect of angiogenesis-related factors on the development and progression of CKD has been examined $[3,4]$. However, most of these studies have focused on proangiogenic factors, such as VEGF-A and angiopoietin-1 [5-8]. VEGF-A, a potent proangiogenic factor, is involved in the development of the kidney [20, $21]$ and also plays an important role in maintaining the glomerular capillary structure and in the repair process following injuries of glomerular endothelial cells and peritubular capillaries $[6,22,23]$. Physiological levels of VEGF-A are also required for maintenance of the glomerular filtration barrier [7]. Angiopoietin-1 enhances endothelial cell survival and stabilization as well as plays 
Table 2. Multivariable-adjusted regression coefficients (95\% CI) of one standard deviation higher in log-transformed plasma endostatin $(0.55 \mathrm{ng} / \mathrm{dl})$ with eGFR and log-transformed urinary albumin

\begin{tabular}{|c|c|c|c|c|}
\hline & \multicolumn{2}{|l|}{$\begin{array}{l}\text { eGFR } \\
\mathrm{ml} / \mathrm{min} / 1.73 \mathrm{~m}^{2}\end{array}$} & \multicolumn{2}{|c|}{$\begin{array}{l}\text { log-transformed albuminuria } \\
\mathrm{mg} / 24 \mathrm{~h}\end{array}$} \\
\hline & effect size (95\% CI) & $\mathrm{p}$ value & effect size $(95 \% \mathrm{CI})$ & $\mathrm{p}$ value \\
\hline Age-, gender-, and race-adjusted & $-26.3(-28.0,-24.5)$ & $<0.0001$ & $1.33(1.18,1.48)$ & $<0.0001$ \\
\hline Multivariable-adjusted ${ }^{1}$ & $-26.2(-28.3,-24.0)$ & $<0.0001$ & $1.19(1.02,1.37)$ & $<0.0001$ \\
\hline Multivariable-adjusted $^{2}$ & $-24.5(-26.8,-22.3)$ & $<0.0001$ & $1.17(0.98,1.36)$ & $<0.0001$ \\
\hline
\end{tabular}

\footnotetext{
${ }^{1}$ Adjusted for age, gender, race, high school education, physical activity, current cigarette smoking, weekly alcohol drinking, BMI, LDL-cholesterol, serum glucose, systolic blood pressure, and history of CVD.

${ }^{2}$ Additionally adjusted for history of hypertension and diabetes.
}

a pivotal role in the maturation of glomeruli and renal blood vessels [24]. Reduction in circulating or renal levels of VEGF-A and angiopoietin-1 has been reported in patients with CKD who had moderate-to-severe renal dysfunction [25].

The inhibitory effects of endostatin on the expression of VEGF-A and angiopoietin-1 and on endothelial cellspecific angiogenesis have been reported in experimental studies $[9,11,26]$. These data suggest that increased endostatin may lead to impaired renal repair $[12,13]$. On the other hand, an animal study has suggested that endostatin may have renoprotective effects in streptozotocin-induced diabetic nephropathy [14]. However, there are very few studies which have investigated the association between circulating endostatin and risk of CKD in human subjects. In the only published clinical study, Futrakul et al. [15] reported that plasma endostatin was increased in 60 Thai patients with moderately impaired kidney function $(356 \pm 126 \mathrm{pg} / \mathrm{ml})$ compared to 15 normal Thai controls $(122 \pm 21 \mathrm{pg} / \mathrm{ml} ; \mathrm{p}<0.001$ for group differences). They also reported significantly decreased levels of VEGF in CKD patients compared to controls [15]. In that pilot study, information on GFR, albuminuria, and other covariables was not reported. Our study was conducted with white and black Americans, and CKD was clearly defined using eGFR and albuminuria. Moreover, multiple covariables for CKD were carefully collected and used for adjustment in the multivariable analyses. Our study not only found that plasma endostatin was significantly increased in patients with CKD, but also discovered an independent and dose-response association between plasma level of endostatin and the severity of CKD measured by eGFR and albuminuria.
Table 3. Odds ratio of CKD associated with the top tertile $(\geq 131.4$ $\mathrm{ng} / \mathrm{dl})$ compared with the two lower tertiles $(<131.4 \mathrm{ng} / \mathrm{dl})$ of plasma endostatin

\begin{tabular}{lll}
\hline & Odds ratio (95\% CI) & p value \\
\hline Age-, gender-, and race-adjusted & $31.0(15.9,60.5)$ & $<0.0001$ \\
Multivariable-adjusted $^{1}$ & $21.6(10.2,45.5)$ & $<0.0001$ \\
Multivariable-adjusted $^{2}$ & $19.1(8.4,43.5)$ & $<0.0001$ \\
\hline
\end{tabular}

${ }^{1}$ Adjusted for age, gender, race, high school education, physical activity, current cigarette smoking, weekly alcohol drinking, BMI, LDL-cholesterol, serum glucose, systolic blood pressure, and history of CVD.

${ }^{2}$ Additionally adjusted for history of hypertension and diabetes.

Several potential limitations of our study should be noted. First, the cross-sectional nature of our study makes it difficult to draw any inferences regarding causality between plasma endostatin and risk of CKD. In addition, other angiogenesis-related factors, such as VEGF-A and angiopoietin-1 were not measured in our study. However, their association with CKD have been reported in previous studies $[7,8,25]$. Finally, our study has a relatively small sample size. A large prospective cohort study might provide more definite evidence for the association of plasma endostatin with CKD.

In conclusion, our study found a strong, independent, and dose-response association between the circulating level of endostatin and severity of CKD. However, its pathogenic role in the development and progression of $\mathrm{CKD}$ remains to be determined. Prospective cohort studies and clinical trials are warranted to further examine 
the causal relationship between endostatin and risk of $\mathrm{CKD}$, and to develop novel interventions targeting endostatin aimed at reducing CKD risk.

\section{Acknowledgements}

The authors would like to express their appreciation to A. Brent Alper, MD; Mahfuz Rahman, MD; Grace Browne, BS; Ji Hua $\mathrm{Xu}, \mathrm{MD}, \mathrm{PhD}$; Nishant B. Jalandhara, MD; Kanwaljit K. Chouhan, MD; Kathryn Royse, MPH; Stephanie Rogers, MPH;
David Mire, MPH; and Lora R. Nolen, MPH, for their assistance in this project. This study was supported by a grant (P20RR017659) from the National Center for Research Resources, National Institutes of Health (Bethesda, Md., USA) and partially supported by a Clinical and Translational Clinical Research Center grant from the Louisiana Board of Regents RC/EEP Fund.

\section{Disclosure Statement}

We have no conflicts of interest to declare.

\section{References}

$\checkmark 1$ Levey AS, Coresh J: Chronic kidney disease. 11 Hajitou A, Grignet C, Devy L, Berndt S, Lancet 2011;379:165-180.

- Kronenberg F: Emerging risk factors and markers of chronic kidney disease progression. Nat Rev Nephrol 2009;5:677-689.

13 Lerman LO, Chade AR: Angiogenesis in the kidney: a new therapeutic target? Curr Opin Nephrol Hypertens 2009;18:160-165.

4 Maeshima Y, Makino H: Angiogenesis and chronic kidney disease. Fibrogenesis Tissue Repair 2010;3:13.

5 Kang DH, Anderson S, Kim YG, Mazzalli M, Suga S, Jefferson JA, Gordon KL, Oyama TT, Hughes J, Hugo C, Kerjaschki D, Schreiner GF, Johnson RJ: Impaired angiogenesis in the aging kidney: vascular endothelial growth factor and thrombospondin-1 in renal disease. Am J Kidney Dis 2001;37:601611.

6 Eremina V, Sood M, Haigh J, Nagy A, Lajoie G, Ferrara N, Gerber HP, Kikkawa Y, Miner JH, Quaggin SE: Glomerular-specific alterations of VEGFA expression lead to distinct congenital and acquired renal diseases. J Clin Invest 2003;111:707-716.

7 Hovind P, Tarnow L, Oestergaard PB, Parving $\mathrm{HH}$ : Elevated vascular endothelial growth factor in type 1 diabetic patients with diabetic nephropathy. Kidney Int Suppl 2000;75:S56-S61.

$\checkmark 8 \mathrm{Kim} \mathrm{NH}, \mathrm{Oh} \mathrm{JH}$, Seo JA, Lee KW, Kim SG, Choi KM, Baik SH, Choi DS, Kang YS, Han SY, Han KH, Ji YH, Cha DR: Vascular endothelial growth factor (VEGF) and soluble VEGF receptor FLT-1 in diabetic nephropathy. Kidney Int 2005;67:167-177.

-9 Yamaguchi N, Anand-Apte B, Lee M, Sasaki T, Fukai N, Shapiro R, Que I, Lowik C, Timpl R, Olsen BR: Endostatin inhibits VEGF-induced endothelial cell migration and tumor growth independently of zinc binding. EMBO J 1999;18:4414-4423.

10 Hanai J, Dhanabal M, Karumanchi SA, Albanese C, Waterman M, Chan B, Ramchandran R, Pestell R, Sukhatme VP: Endostatin causes G1 arrest of endothelial cells through inhibition of cyclin D1. J Biol Chem 2002; 277:16464-16469.
Blacher S, Deroanne CF, Bajou K, Fong T, Chiang Y, Foidart JM, Noel A: The antitumoral effect of endostatin and angiostatin is associated with a down-regulation of vascular endothelial growth factor expression in tumor cells. FASEB J 2002;16:1802-1804.

12 O'Riordan E, Mendelev N, Patschan S, Patschan D, Eskander J, Cohen-Gould L, Chander P, Goligorsky MS: Chronic NOS inhibition actuates endothelial-mesenchymal transformation. Am J Physiol Heart Circ Physiol 2007;292:H285-H294.

13 Stoessel A, Paliege A, Theilig F, Addabbo F, Ratliff B, Waschke J, Patschan D, Goligorsky MS, Bachmann S: Indolent course of tubulointerstitial disease in a mouse model of subpressor, low-dose nitric oxide synthase inhibition. Am J Physiol Renal Physiol 2008; 295:F717-F725.

14 Ichinose K, Maeshima Y, Yamamoto Y, Kitayama H, Takazawa Y, Hirokoshi K, Sugiyama H, Yamasaki Y, Eguchi K, Makino H: Antiangiogenic endostatin peptide ameliorates renal alterations in the early stage of a type 1 diabetic nephropathy model. Diabetes 2005; 54:2891-2903.

15 Futrakul N, Butthep P, Laohareungpanya N, Chaisuriya P, Ratanabanangkoon K: A defective angiogenesis in chronic kidney disease. Ren Fail 2008;30:215-217.

16 Pickering TG, Hall JE, Appel LJ, Falkner BE, Graves J, Hill MN, Jones DW, Kurtz T, Ships SG, Rochelle EJ: Recommendations for blood pressure measurement in humans and experimental animals: part 1: blood pressure measurement in humans. Circulation 2005; 111:697-716

17 Verbraecken J, Van de Heyning P, De Backer $\mathrm{W}$, Van Gaal L: Body surface area in normalweight, overweight, and obese adults. A comparison study. Metabolism 2006;55:515-524.
8 Levey AS, Cores J, Greene T, Lesley AS, Zhang Y, Hendrickson S, Cusec JW, Van Lenten F, for the Chronic Kidney Epidemiology Collaboration: Using standardized serum keratinize values in the modification of diet in renal disease study equation for estimating glomerular filtration rate. Ann Intern Med 2006;145:247-254.

19 McGreevy KM, Lipsitz SR, Linder JA, Rimm E, Hoel DG: Using median regression to obtain adjusted estimates of central tendency for skewed laboratory and epidemiologic data. Clin Chem 2009;55:165-169.

20 Kitamoto Y, Tokunaga H, Tomita K: Vascular endothelial growth factor is an essential molecule for mouse kidney development: glomerulogenesis and nephrogenesis. J Clin Invest 1997;99:2351-2357.

21 Tufro A, Norwood VF, Carey RM, Gomez RA: Vascular endothelial growth factor induces nephrogenesis and vasculogenesis. J Am Soc Nephrol 1999;10:2125-2134.

-22 Masuda Y, Shimizu A, Mori T, Ishiwata T, Kitamura H, Ohashi R, Ishizaki M, Asano G, Sugisaki Y, Yamanaka N: Vascular endothelial growth factor enhances glomerular capillary repair and accelerates resolution of experimentally induced glomerulonephritis. Am J Pathol 2001;159:599-608.

23 Shimizu A, Masuda Y, Mori T, Kitamura H, Ishizaki M, Sugisaki Y, Fukuda Y: Vascular endothelial growth factor165 resolves glomerular inflammation and accelerates glomerular capillary repair in rat anti-glomerular basement membrane glomerulonephritis. J Am Soc Nephrol 2004;15:2655-2665.

24 Woolf AS, Yuan HT: Angiopoietin growth factors and Tie receptor tyrosine kinases in renal vascular development. Pediatr Nephrol 2001;16:177-184

25 Futrakul N, Butthep P, Futrakul P: Altered vascular homeostasis in chronic kidney disease. Clin Hemorheol Microcirc 2008;38: 201-207.

26 Yokoyama Y, Ramakrishnan S: Improved biological activity of a mutant endostatin containing a single amino-acid substitution. Br J Cancer 2004;90:1627-1635. 